\title{
Maximum-Likelihood Classification for MPSK with Compressive Samplings
}

\author{
Tong Nian ${ }^{1, a}$, Li Lichun ${ }^{1, b}$ and Lu Xun ${ }^{1, c}$ \\ ${ }^{1}$ PLA Information Engineering University, China

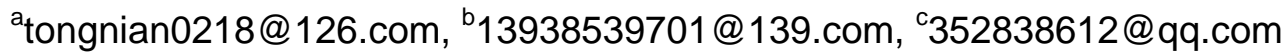

Keywords: modulation classification; MPSK; compressive sensing

\begin{abstract}
This paper focuses on the classification of the MPSK modulations using compressive measurements in additional Gaussian white noise (AWGN). Under the compressive sensing (CS) frame, the compressive maximum-likelihood (CML) classifier provided in this paper tries to recognize the MPSK signals using far fewer samplings than traditional maximum-likelihood (TML) classifier needs. This paper presents the criterion of classification and the classification performance analysis. Finally, several numerical simulations are provided and the results indicate that the CML classifier have a satisfied performance in higher SNR with far lower complexity. It's an effective approach to promote the real-time property of communication system.
\end{abstract}

\section{Introduction}

A new framework called compressive sensing for simultaneous sensing and compression has developed recently. A potentially large reduction can be realized in the sampling and computation costs for a communication system under CS framework. Candès ${ }^{[1]}$, Tao ${ }^{[2]}$ and Donoho ${ }^{[3]}$ present CS in 2006. CS specifies that a signal having a spare representation in one basis can be reconstructed from a small set of projections. The measurement basis is incoherent with the first basis. The CS measurement process is nonadaptive and the reconstruction process is nonlinear. CS has many promising applications in signal acquisition, compression, medical imaging, and sensor networks.

In the modern communication system, modulation classification is an important step between the signal receiving and the signal demodulation. Especially in the non-cooperative communication systems, modulation classification plays a more significant role which can identify the modulation type of a modulated signal corrupted by noise. Many methods have been used in the modulation classification, such as spectrum, moments, zero crossings and Bayes decision theory ${ }^{[5]}{ }^{[6]}$.

While the CS literature has focused almost exclusively on problems in signal reconstruction or approximation, this is frequently not necessary. For instance, in many signal processing applications (including most communications and many radar systems), signals are acquired only for the purpose of making a detection or classification decision. Our aim in this paper is to show that the CS framework is useful for a much wider range of statistical inference tasks. Tasks such as classification do not require a reconstruction of the signal, but only require estimates of the relevant sufficient statistics for the problem. The key finding is that in many cases it is possible to directly extract these statistics from a small number of random projections without ever reconstructing the signal. This work expands on the previous work on classification using compressive measurements.

The papers on the modulation classification under CS frame is not too many ${ }^{[7][8]}$.In this paper, based on the Bayes decision theory, we use the compressive measurements to recognize the modulation type of signals directly. In the framework of CS, modulation classification can process far less data than the conventional approach with the Nyquist sampling rate, so that the speed of modulation classification will be faster and the real-time performance of the communication system can be promoted significantly. 


\section{Background of Compressive Sensing}

Sparse representation of signal. If a few entries of a signal are zero or close to zero, this signal is sparse or compressible. Generally, a signal in time domain is always not sparse. However, in some other domains, the signal may be represented sparsely. Consider a finite-length, one-dimensional, discrete-time signal $x$ which can be represented by a vector with elements $x[n]$ in $\mathbf{R}^{\mathrm{N}}, \quad n=1,2, \ldots, \mathrm{N}$. And a basis $\boldsymbol{\Psi}$ consists of vectors $\psi_{i}$ which has $\mathrm{N}$ entries , $i=1,2, \ldots \mathrm{N}$. So the signal $\boldsymbol{x}$ can be expressed in the basis $\boldsymbol{\Psi}$ as follows:

$$
x=\Psi s
$$

where $\boldsymbol{s}$ is the $\mathrm{N} \times 1$ column vector of weighting coefficients $s_{i}=<\boldsymbol{x}, \boldsymbol{\psi}_{i}>$. $\boldsymbol{s}$ is the representation of signal $\boldsymbol{x}$ in basis $\boldsymbol{\Psi}$. If the weighting coefficients $\boldsymbol{s}$ has only K entries non-zero, the signal $\boldsymbol{x}$ is $\mathrm{K}$-sparse in basis $\boldsymbol{\Psi}$.

Compressive Sensing. Consider a $1 \times$ M measurement vector $\boldsymbol{y}$ with $\mathrm{M}$ entries $y[m], m=1,2, \ldots \mathrm{M}$. A $\mathrm{M} \times \mathrm{N}(\mathrm{M}<<\mathrm{N})$ measurement matrix $\boldsymbol{\Phi}$ can be viewed as $\left[\phi_{1}, \phi_{2}, \ldots, \phi_{\mathrm{M}}\right]^{T}$, and $\phi_{j}, j=1,2, \ldots, \mathrm{M}$ is a $1 \times M$ vector. $y[j]$ is generated by the inner production of $x$ and $\phi_{j}$, so that $y$ can be written as

$$
\boldsymbol{y}=\boldsymbol{\Phi} \boldsymbol{x}=\boldsymbol{\Phi} \Psi \boldsymbol{s}=\Theta s
$$

where $\boldsymbol{\Theta}$ is a $\mathrm{M} \times \mathrm{N}$ matrix that $\boldsymbol{\Theta}=\boldsymbol{\Phi} \boldsymbol{\Psi}$. From [1][2], the measurement process is non-adaptive, because the matrix $\boldsymbol{\Phi}$ is a fixed matrix which does not depend on signal $\boldsymbol{x}$.

\section{MPSK Classification With Compressive Maximum-Likelihood Classifier}

MPSK signal model. The modulation of phase-shift keying uses the phase information of a signal during symbols to carry information, so that the signal can be better transmitted in noise environments. This kind of modulated signal can be expressed as ${ }^{[10]}$ :

$$
\begin{gathered}
\tilde{x}_{l}[n]=\mathrm{A} \cos \left(2 \pi f_{c} n+\theta_{c}+\theta_{l}\right) \\
l \mathrm{~K}<n \leq(l+1) \mathrm{K}, l=0,1,2, \ldots \\
\boldsymbol{x}=\sum_{l=1}^{\mathrm{L}} \boldsymbol{x}_{l}
\end{gathered}
$$

where $\mathrm{A}$ is the signal amplitude, $f_{c}$ and $\theta_{c}$ are the carrier frequency and phase. $\mathrm{K}$ is the number of signal samplings during a symbol period. $\tilde{\boldsymbol{x}}_{l}$ means the signal during $l$ th symbol, i.e. $\tilde{\boldsymbol{x}}_{l}=[x[(l-1) \mathrm{K}+1], x[(l-1) \mathrm{K}+2], \ldots, x[l \mathrm{~K}]]^{T} . \boldsymbol{x}_{l}$ is a $\mathrm{N} \times 1$ vector that fills with $\tilde{\boldsymbol{x}}_{l}$ in the $((l-1) \mathrm{K}+1)$-th to $l \mathrm{~K}$-th entries and fills zero in other entries, i.e. $\boldsymbol{x}_{l}=[0,0, \ldots, 0, x[(l-1) \mathrm{K}+1], x[(l-1) \mathrm{K}+2], \ldots, x[l \mathrm{~K}], 0,0, \ldots, 0]^{T}$. And $\theta_{l}$ is the modulated phase which carries the information of the signal in the $l$-th symbol.

Compressive maximum-likelihood classifier. Instead of the samplings of $r$ observed in the TMC classifier, the measurements $\boldsymbol{y}=\boldsymbol{\Phi}(\boldsymbol{x}+\boldsymbol{v})$ are what we observe, where $\boldsymbol{\Phi}$ is a $\mathrm{M} \times \mathrm{N}(\mathrm{M}<\mathrm{N})$ measurement matrix.

Compared with C hypotheses considered in the last section, the compressive maximum-likelihood classifier assumes that:

$$
\begin{gathered}
\tilde{H}_{j}: \boldsymbol{y}=\boldsymbol{\Phi}(\boldsymbol{x}+\boldsymbol{v})=\boldsymbol{\Phi}\left(\sum_{l=1}^{\mathrm{L}} \boldsymbol{x}_{l}\right)+\boldsymbol{\Phi} \boldsymbol{v} \\
j=1,2, \ldots, \mathrm{C}
\end{gathered}
$$

where $\boldsymbol{x}$ is modulated by $\Delta_{j}$, and $\boldsymbol{y}$ is the $\mathrm{M} \times 1$ vector of measurements.

The expectation of $y$ can be calculated as:

$$
E[\boldsymbol{y}]=E[\boldsymbol{\Phi}(\boldsymbol{x}+\boldsymbol{v})]=E\left[\boldsymbol{\Phi}\left(\sum_{l=1}^{\mathrm{L}} \boldsymbol{x}_{l}\right)+\boldsymbol{\Phi} \boldsymbol{v}\right]=\boldsymbol{\Phi} \boldsymbol{x}
$$

The variance of ${ }^{\boldsymbol{y}}$ can be calculated as: 


$$
D[\boldsymbol{y}]=E\left[(\boldsymbol{y}-E[\boldsymbol{y}])(\boldsymbol{y}-E[\boldsymbol{y}])^{T}\right]=E\left[\boldsymbol{\Phi} \boldsymbol{v}^{T} \boldsymbol{\Phi}^{T}\right]=\sigma^{2} \boldsymbol{\Phi} \boldsymbol{\Phi}^{T}
$$

Considered with the expectation and variance of ${ }^{\boldsymbol{y}}$, the $\operatorname{PDF}{ }^{\boldsymbol{y}}$ is:

$$
\begin{gathered}
f_{j}(\boldsymbol{y})=\frac{\exp \left(-\frac{1}{2}(\boldsymbol{y}-\boldsymbol{\Phi} \boldsymbol{x})^{T}\left(\sigma^{2} \boldsymbol{\Phi} \boldsymbol{\Phi}^{T}\right)^{-1}(\boldsymbol{y}-\boldsymbol{\Phi} \boldsymbol{x})\right)}{\left|\sigma^{2} \boldsymbol{\Phi} \boldsymbol{\Phi}^{T}\right|^{\frac{1}{2}}(2 \pi)^{\frac{\mathrm{M}}{2}}} \\
f_{j}(\boldsymbol{y}) \propto \exp \left(\boldsymbol{y}^{T}\left(\boldsymbol{\Phi} \boldsymbol{\Phi}^{T}\right)^{-1} \boldsymbol{\Phi} \boldsymbol{x}\right)
\end{gathered}
$$

The compressive maximum likelihood function can be built as:

$$
\tilde{\mathcal{I}}_{j}=\ln \left(\prod_{l=1}^{\mathrm{L}} E_{\theta_{l}}\left[\exp \left(\boldsymbol{y}^{T}\left(\boldsymbol{\Phi} \boldsymbol{\Phi}^{T}\right)^{-1} \boldsymbol{\Phi} \boldsymbol{x}_{l}\right)\right]\right)=\sum_{l=1}^{\mathrm{L}} \ln \left\{\frac{1}{Z_{j}} \sum_{i=1}^{Z_{j}} \exp \left(\boldsymbol{y}^{T}\left(\boldsymbol{\Phi} \boldsymbol{\Phi}^{T}\right)^{-1} \boldsymbol{\Phi} \boldsymbol{x}_{l, i}\right)\right\}
$$

The maximum-likelihood classifier chooses the hypotheses whose maximum likelihood function is the maximized:

$$
\tilde{H}_{j}^{*}=\arg \max _{H_{j}} \tilde{\mathcal{I}}_{j}
$$

\section{Simulation Results}

In the following experiments, the modulations recognition will take place from the set $\{B P S K, Q P S K, 8 P S K\}$, and the results of figure 1 and figure 2 are based on 500 Monte Carlo trials during 40 symbols. SNR in decibels is defined as:

$$
S N R=10 \log _{10}\left(\frac{E_{s}}{\sigma^{2}}\right)
$$

where, $E_{s}$ is the energy of a symbol, $\sigma^{2}$ is the noise variance.

In figure1and figure2, we calculate the classification probability of BPSK, QPSK and 8PSK using different algorithms and different measurements respectively.

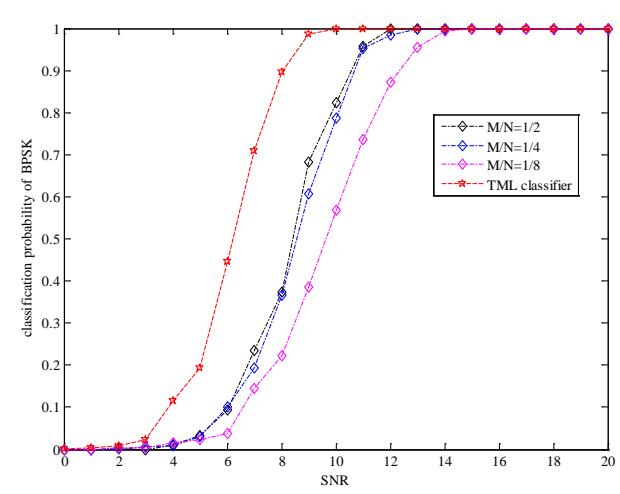

Fig.1 Effect of $M$ and SNR on classification probability of BPSK

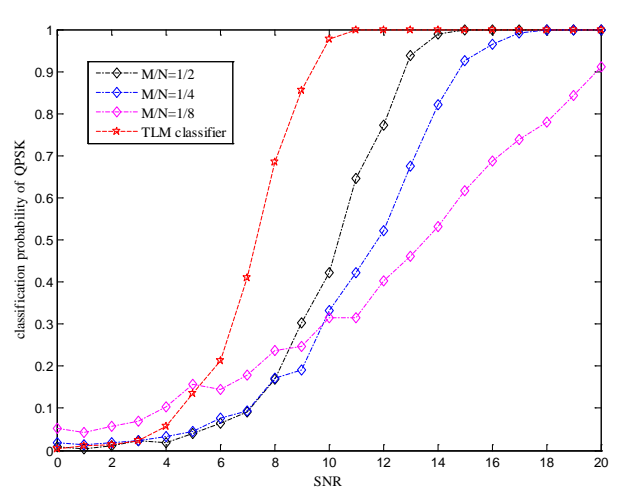

Fig. 2 Effect of $M$ and SNR on classification probability of QPSK

In addition, we compare the classification performance with different measurements and SNR. The probability of classification of CML classifier depends on the SNR and the number of measurements. The performance of the compressive classifier will be better in higher SNR with more measurements. We can also find that in high SNR, the classification probability can be very high with very few measurements.

\section{Summary}

In this paper, the problem of MPSK modulation classification is discussed. Instead of TML 
classifier, we present a new kind of classifier-CML classifier under the frame of compressive sensing. We have derived the compressive maximum likelihood function and analyze the error probability of compressive classifier.

The simulation results show that the classification probability is related on SNR and the number of compressive measurements. And the 8PSK classification performance is better than QPSK and BPSK. What's more, it's important to design an appropriate measurement matrix to achieve a perfect performance.

\section{References}

[1] Candès E, Rom B J. Quantitative robust uncertainty principles and optionally sparse decompositions [J]. Foundations of Compute Math, 2006, 6(2): 227-254.

[2] Candès E, Tao T. Near optional signal recovery from random projections Universal encoding strategies [J]. IEEE Transactions on Information Theory, 2006, 52(12): 5406-5425.

[3] Donohd D L. Compressed Sensing [J]. IEEE Transactions on Information Theory, 2006 , 52(4): 1289-1306.

[4] Davenport M A, Wakin M B, Baraniuk R G. Detection and estimation with compressive measurements [R]. Technical Report TREE 0610, November 2006.

[5] Dobre O A, Abdi A, Bar-Ness Y, Su W. Survey of automatic modulation classification techniques: classical approaches and new trends [J]. Communications, IET, vol.1, no.2, pp.137-156, April 2007.

[6] Zhao Zhijin, Lang Tao. A MPSK modulation classification method based on the maximum likelihood criterion [J]. Signal Processing, 2004. Proceedings. ICSP '04. 2004 7th, vol. 2, pp.1805 1808.

[7] Chia Wei Lim and Wakin M B. Automatic modulation recognition for spectrum sensing using nonuniform compressive samples [C]. IEEE International Conference on Communications (ICC), June 10-15, 2008, Ottawa, Canada, 2012: 3505 - 3510.

[8] Haupt J, Castro R, Nowak R, G Fudge, et al. Compressive Sampling for Signal Classification. Proc. 40th Asilomar Conf. on Signals, Systems, and Computers, Oct. 29 - Nov. 1, 2006, Pacific Grove, California, USA, 2006: 1430-1434.

[9] Kay S M. Fundamentals of Statistical Signal Processing, Volume 2: Detection Theory, Prentice Hall, 1998.

[10] Wei W, Mendel J M. Maximum-likelihood classification for digital amplitude-phase modulations[J]. IEEE Trans. Commun., 2000, 48, pp. 189- 193. 